\title{
Bulk reconstruction in AdS and Gel'fand-Graev-Radon transform
}

\author{
Samrat Bhowmick, ${ }^{a}$ Koushik Ray ${ }^{a}$ and Siddhartha Sen ${ }^{b}$ \\ ${ }^{a}$ Department of Theoretical Physics, Indian Association for the Cultivation of Science, \\ Kolkata 700 032, India \\ ${ }^{b}$ CRANN, Trinity College Dublin, \\ Dublin - 2, Ireland \\ E-mail: tpsb5@iacs.res.in, koushik@iacs.res.in, sen1941@gmail.com
}

ABSTRACT: The bulk reconstruction formula for a Euclidean anti-de Sitter space is directly related to the inverse of the Gel'fand-Graev-Radon transform. Correlation functions of a conformal scalar field theory in the boundary are thereby related to correlation functions of a self-interacting scalar field theory in the bulk at different loop orders.

KEYwords: AdS-CFT Correspondence, Differential and Algebraic Geometry

ARXIV EPrinT: 1705.06985 
Holographic duality is a correspondence relating a quantum field theory on a given spacetime, referred to as the bulk, to one on its boundary, possibly in different ranges of couplings. The conjectured duality between a theory of closed strings in the $n$-dimensional anti-de Sitter space as the bulk and a conformal field theory on the boundary is an example of holographic duality [1-3]. It relates observables in the bulk anti-de Sitter space at weak coupling to correlation functions of a strongly coupled theory on the boundary and vice versa. The symmetries of a dual pair thus related ensure that both theories have the same number of degrees of freedom, although the scheme of organizing them into fields are rather different in the two theories. While the duality is expected to work both ways as an equivalence relation, in practice, obtaining the theory in a given bulk from a given theory on the boundary appears to be more difficult. This is known as the problem of bulk-reconstruction.

Within the scope of scalar field theories both in the bulk and on the boundary, to which we restrict ourselves in this note, a bulk-reconstruction procedure has been invented [4-17]. While the normalizable modes of the bulk scalar fields are identified with operators of the conformal field theory on the boundary, the latter produce bulk fields via an integral kernel, called the smearing function.

In this note we relate the bulk reconstruction problem to integral geometry. A scalar field on the boundary is taken to be the Gel'fand-Graev-Radon transform of a scalar field in the anti-de Sitter space [18]. This is a generalization of the Radon transform on Euclidean spaces to Lobachevskian spaces. Integral geometry studies the problem of determination of a function on a manifold from the integral of the function on a family of submanifolds. For the anti-de Sitter space, realized through a quadratic form in the Euclidean space of one higher dimension, a suitable choice of the submanifolds is obtained via the null cone. The boundary is obtained as a limiting submanifold of the family. We show that if the Gel'fand-Graev-Radon transform of a function possesses certain scaling properties on the null cone, then it can be used to write the function in the anti-de Sitter space from its integral on the boundary. Distributions or fields can be treated similarly.

The invertible Gel'fand-Graev-Radon transform induces a transform between scalar field actions in the bulk and on the boundary, namely, the two actions, while expressed in terms of different fields, are numerically equal. We obtain the induced action on the boundary corresponding to a self-interacting scalar field theory in the $n$-dimensional bulk, in particular, a $\phi^{k}$-theory. The construction guarantees that the theory on the boundary is conformal. Writing the corresponding generating functionals then leads to relating correlation functions of the two theories.

Let us start by recalling some aspects of the Gel'fand-Graev-Radon (GGR) transform. The most studied arena for Radon transform is manifolds of constant curvature. Distributions on Grassmannian submanifolds of different codimensions are obtained through the Radon transform, which can then be inverted [19]. One example of such submanifolds is the set of geodesics [20]. We shall restrict to another one-dimensional submanifold, the set of lines through the origin. We begin with a discussion of some features of it to be used 


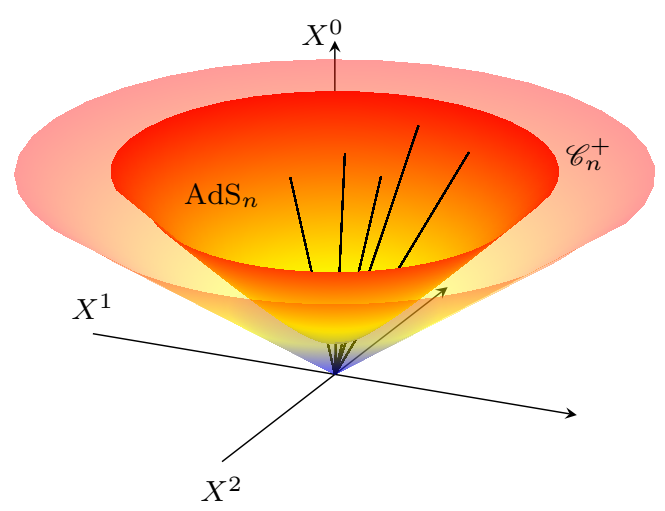

Figure 1. Null cone and $\operatorname{AdS}_{n}$

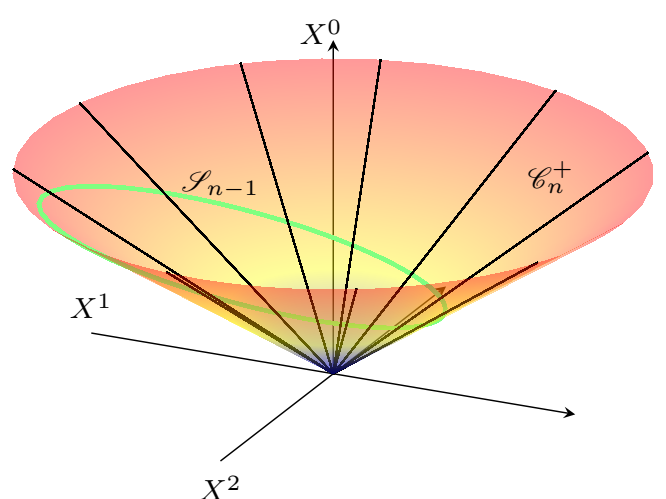

Figure 2. Null cone and $\mathscr{S}_{n-1}$.

here. Let $\mathcal{M}$ denote the $n$-dimensional Lobachevskian space or the Euclidean anti-de Sitter space. In this note $n>1$. In the coordinates $\left\{X^{a} \mid a=0, \cdots, n\right\}$ of the $(n+1)$-dimensional affine space $\mathbf{R}^{n+1}$ with metric $\eta_{a b}=\operatorname{diag}(-1,1, \cdots, 1), \mathcal{M}$ is defined as the hypersurface

$$
\eta_{a b} X^{a} X^{b}=-L^{2}
$$

where $L$ is a real number. An equivalent description of $\mathcal{M}$ is as the set of straight lines passing through the origin of $\mathbf{R}^{n+1}$ within the region

$$
\eta_{a b} X^{a} X^{b}<0 .
$$

The isomorphism between these two stems from the fact that each of these lines intersects the hypersurface (1) at a single point. This is depicted in figure 1 for $n=2$. We shall also consider the $n$-dimensional positive null cone $\mathscr{C}_{n}^{+}$defined as the set of null vectors $\xi^{a}$ in $\mathbf{R}^{n+1}$, that is,

$$
\eta_{a b} \xi^{a} \xi^{b}=0, \quad \xi^{0} \geqslant 0
$$

also shown in figure 1 . For a point $X$ in $\mathcal{M}$ and a point $\xi$ in $\mathscr{C}_{n}^{+}$, let us consider the family of hypersurfaces $\mathscr{S}_{n-1}(p)$, given by

$$
\Sigma:=\eta_{a b} X^{a} \xi^{b}+p=0
$$

where $p$ is a real parameter and $X^{a}$ and $\xi^{a}$ satisfy (1) and (3), respectively. For a fixed non-zero $p$ this is called a horosphere [18]. The only solution for $X$ when $p$ vanishes is $X^{a}=\xi^{a}$, points on the cone. Looked upon as a subspace of the cone, this is depicted in figure 2. Every line on the cone passing through the apex intersects $\mathscr{S}_{n-1}$ only once. The hypersurface $\mathscr{S}_{n-1}$ can alternatively viewed as a subspace of $\mathcal{M}$. Using the isomorphism of $\mathcal{M}$ with the lines through the origin, these two descriptions coincide as $X^{0} \longrightarrow \infty$. Hence the boundary of $\mathcal{M}$ falls on the cone $\mathscr{C}_{n}^{+}$. For a point $\xi$ on the cone $\mathscr{C}_{n}^{+}$, the GGR transform of a smooth function $f$ with bounded support on $\mathcal{M}$ is given by the integral

$$
h_{p}(\xi)=\int_{\mathcal{M}} f(X) \delta\left(\eta_{a b} X^{a} \xi^{b}+p\right) d^{n} X
$$


where $d^{n} X=\frac{d X^{1} d X^{2} \ldots d X^{n}}{X^{0}}$ is the volume element on $\mathcal{M}$ induced by (1). The inverse transform, when exists, yields a function at a point in $\mathcal{M}$ from a function on the light cone as

$$
f(X)=c_{n} \int_{\mathscr{C}_{n}^{+}} \frac{h_{p}(\xi)}{\left|\eta_{a b} X^{a} \xi^{b}+p\right|^{n}} d^{n} \xi
$$

where $c_{n}$ is a constant, depending on the dimension $n$ and $d^{n} \xi$ denotes the volume element on the null cone $\mathscr{C}_{n}^{+}$induced by (3), namely,

$$
d^{n} \xi=\frac{1}{\xi^{n}} d \xi^{0} \cdots d \xi^{n-1} .
$$

Consistency of (5) and (6) requires

$$
c_{n} \int_{\mathscr{C}_{n}^{+}} \frac{\delta\left(\eta_{a b} X^{a} \xi^{b}+p\right)}{\left|\eta_{a b} Y^{a} \xi^{b}+p\right|^{n}} d^{n} \xi=\delta_{\mathcal{M}}(X-Y),
$$

where $\delta_{\mathcal{M}}(X-Y)$ denotes the delta function on $\mathcal{M}$. The constant $c_{n}$ is determined from the normalization of the delta function as

$$
c_{n}=2 L \alpha^{2} \frac{\sin \frac{n \pi}{2}}{(2 \sqrt{\pi})^{n-1}} \frac{\Gamma(n)}{\Gamma\left(\frac{n+1}{2}\right)},
$$

where we have introduced a dimension-less constant $\alpha=p / L$. Computation of the constant is relegated to the end.

The GGR transform (5) of a function and its inverse (6) pertain to $\mathcal{M}$ and the null cone. In order to relate it to the bulk reconstruction we need to first specify the boundary of $\mathcal{M}$ and relate it to the null cone. Let us consider an affine chart on $\mathcal{M}$,

$$
\begin{aligned}
X^{0} & =\frac{z L}{2}\left(1+\frac{1+x^{2}}{z^{2}}\right), \quad X^{i}=\frac{x^{i} L}{z}, \quad X^{n}=\frac{z L}{2}\left(1-\frac{1-x^{2}}{z^{2}}\right), \\
x^{2} & =\sum_{i=1}^{n-1}\left(x^{i}\right)^{2}, \quad-\infty<x^{i}<\infty, 0 \leqslant z<\infty ; \quad i=1, \cdots, n-1 .
\end{aligned}
$$

These solve (1). The metric $g$ on $\mathcal{M}$ is then given by

$$
d s^{2}=\frac{L^{2}}{z^{2}}\left(d z^{2}+\sum_{i=1}^{n-1}\left(d x^{i}\right)^{2}\right),
$$

the resulting volume element being

$$
d^{n} X=\sqrt{g} d z d^{n-1} x=\frac{L^{n}}{z^{n}} d z d^{n-1} x .
$$

In this chart the boundary $\mathcal{B}_{n-1}$ that we shall be concerned about is located at $z=0$, which in turn leads to $X^{0} \longrightarrow \infty$. The null cone $\mathscr{C}_{n}^{+}$is a metric cone $\mathbf{R}_{+} \times \xi^{0} \mathbf{S}^{n-1}$ over an $(n-1)$-dimensional sphere $\mathbf{S}^{n-1}$ with chart

$$
\begin{aligned}
\xi^{i} & =\frac{2 \tilde{x}^{i}}{1+\tilde{x}^{2}} \xi^{0}, \quad \xi^{n}=-\frac{1-\tilde{x}^{2}}{1+\tilde{x}^{2}} \xi^{0} \\
\tilde{x}^{2} & =\sum_{i=1}^{n-1}\left(\tilde{x}^{i}\right)^{2}, \quad-\infty<x^{i}<\infty, 0 \leqslant \xi^{0}<\infty ; \quad i=1, \cdots, n-1 .
\end{aligned}
$$


These solve (3). In this chart the volume element (7) takes the form

$$
d^{n} \xi=\frac{2^{n-1}\left(\xi^{0}\right)^{n-2}}{\left(1+\tilde{x}^{2}\right)^{n-1}} d \xi^{0} d^{n-1} \tilde{x} .
$$

Substituting (10) and (14) in (4) we obtain the equation for $\mathscr{S}_{n-1}(p)$ in terms of the affine coordinates as

$$
\left(z^{2}+\sum\left(x^{i}-\tilde{x}^{i}\right)^{2}\right) \xi^{0} L=z p\left(1+\sum\left(\tilde{x}^{i}\right)^{2}\right) .
$$

In the limit $\xi^{0} \longrightarrow \infty$ and $z \longrightarrow 0$, this leads to $x^{i} \longrightarrow \tilde{x}^{i}$, describing the boundary. Let us note that vanishing of $p$ results in $c_{n}=0$, by (9), consistent with the fact that there is no "bulk" in this limit. In the following, we shall consider integration on the cone, as in (6). For such purposes, it is important to observe that the sphere at the base of the cone admits smooth deformation to the hypersurface given by a constant value of $\xi^{0}$ and so does the boundary $\mathcal{B}_{n-1}$ too.

Let us assume that the previous considerations hold good for quantum fields. Let $\tilde{\phi}(\tilde{x})$ be a conformal field of dimension $\Delta$ on $\mathcal{B}_{n-1}$, whose coordinates are taken to be $\tilde{x}$. Then,

$$
\tilde{\phi}(\lambda \tilde{x})=\lambda^{-\Delta} \tilde{\phi}(\tilde{x}),
$$

where $\lambda=\lambda(\tilde{x})$ is any function on $\mathcal{B}_{n-1}$. Let us assume that the function (5) is given by the conformal field as [21]

$$
\begin{aligned}
h_{p}(\xi) & =h_{p}\left(\xi^{0}, \cdots, \xi^{n-1}\right) \\
& \stackrel{\text { def }}{=} \tilde{\phi}\left(\frac{2 \tilde{x}^{1}}{1+\tilde{x}^{2}} \xi^{0}, \frac{2 \tilde{x}^{2}}{1+\tilde{x}^{2}} \xi^{0}, \cdots, \frac{2 \tilde{x}^{n-1}}{1+\tilde{x}^{2}} \xi^{0}\right) \\
& =\left(\frac{2 \xi^{0}}{1+\tilde{x}^{2}}\right)^{-\Delta} \tilde{\phi}(\tilde{x}),
\end{aligned}
$$

where we have used (14) at the second step. Inserting this and (16) in (6) we obtain

$$
\phi(z, x)=\phi_{0}(n, \Delta) \int \mathcal{K}(z, x \mid \tilde{x}) \tilde{\phi}(\tilde{x}) d^{n-1} \tilde{x},
$$

with

$$
\mathcal{K}(z, x \mid \tilde{x})=\left(\frac{z^{2}+\sum\left(x^{i}-\tilde{x}^{i}\right)^{2}}{z}\right)^{\Delta+1-n},
$$

where we denoted the field in $\mathcal{M}$ as $f(X)=\phi(z, x)$. The constant $\phi_{0}(n, \Delta)$ is given by

$$
\phi_{0}(n, \Delta)=\frac{2^{n-1-\Delta} c_{n}}{\alpha^{1+\Delta} L^{n}} \int_{0}^{\infty} \frac{\zeta^{n-2-\Delta}}{|1-\zeta|^{n}} d \zeta
$$

where we have defined

$$
\zeta=\left(\frac{\xi^{0}}{z \alpha}\right) \frac{z^{2}+(x-\tilde{x})^{2}}{1+\tilde{x}^{2}} .
$$

The integral in $\zeta$ can be evaluated using the formula

$$
\int_{0}^{\infty} \frac{x^{a}}{(1-x)^{n}} d x=\frac{\Gamma(a+1) \Gamma(1-n)}{\Gamma(a-n+2)},
$$


which, in turn, can be obtained by writing the denominator of the integrand as a Barnes' integral. This yields

$$
\phi_{0}(n, \Delta)=\frac{2^{n-\Delta-1} c_{n}}{\alpha^{\Delta+1} L^{n}} \frac{\pi}{\sin n \pi} \frac{\Gamma(\Delta+1)}{\Gamma(n) \Gamma(\Delta+2-n)}\left(1+(-1)^{n}\right) .
$$

Both the expression for $\phi_{0}$ and the formula (24) are singular, as written, since $n$ is an integer and $n>1$. In order to obtain the normalized GGR transform these are to be evaluated in a regularized manner. Using the formula $\Gamma(z) \Gamma(1-z)=\pi / \sin \pi z$, plugging in the value of $c_{n}$ from (9) and further using the regularized expression

$$
\left(1+(-1)^{n}\right) \frac{\sin \frac{n \pi}{2}}{\sin n \pi}=e^{i n \pi / 2}
$$

we finally obtain $\phi_{0}(n, \Delta)=\Phi_{0}(n, \Delta) / L^{n-1}$, with

$$
\Phi_{0}(n, \Delta)=\frac{e^{i n \pi / 2}}{(2 \alpha)^{\Delta-1} \pi^{(n-3) / 2}} \frac{\Gamma(\Delta+1)}{\Gamma(\Delta-n+2) \Gamma\left(\frac{n+1}{2}\right)} .
$$

Using this expression in (20) gives the formula for bulk reconstruction of a conformal field of scaling dimension $\Delta$ from the boundary of an anti-de Sitter space of dimension $n$ [6]. The expression (26) requires qualification. The inversion of the Radon transform is a wellknown ill-posed problem. It involves computing integrals with prescribed regularization to determine the constant $c_{n}$ [18]. In the present case, the assumption of conformality, (19) brings in factors which conspire to cancel the singularities, yielding the non-singular expression (26).

The bulk field $\phi$ expressed as the inverse transform (20) when operated on by the Laplacian $\square_{\mathcal{M}}$ on $\mathcal{M}$ obeys the equation

$$
\square_{\mathcal{M}} \phi(z, x)=\frac{1}{\sqrt{g}} \partial_{\mu}\left(\sqrt{g} g^{\mu \nu} \partial_{\nu} \phi\right)=\frac{\Delta(\Delta-n+1)}{L^{2}} \phi(z, x),
$$

implying that the scalar field $\phi$ is massive, with mass $m$ given by

$$
m^{2}=\Delta(\Delta-n+1) / L^{2} .
$$

Now that we have obtained the bulk reconstruction formula as a transform which is invertible, we can use it to induce actions from the bulk to the boundary and vice versa. For example, using the metric (12), the action of a free scalar field in $\mathcal{M}$ is

$$
S(\phi)=\int d^{n-1} x d z \sqrt{g}\left[g^{\mu \nu} \partial_{\mu} \phi(z, x) \partial_{\nu} \phi(z, x)+m^{2} \phi^{2}(z, x)\right] .
$$

Plugging in (20) with (27) in this action we obtain the action on the boundary. From the first term of the (30), we obtain

$$
\tilde{S}(\tilde{\phi})=S(\phi)=\frac{\Phi_{0}^{2}(\Delta+1-n)^{2}}{L^{n}} \int \mathcal{P}\left(\tilde{x}, \tilde{x}^{\prime}\right) \tilde{\phi}(\tilde{x}) \tilde{\phi}\left(\tilde{x}^{\prime}\right) d^{n-1} \tilde{x} d^{n-1} \tilde{x}^{\prime},
$$


where $\mathcal{P}$ involves integrations over $z$ and $x^{i}, i=1, \cdots, n-1$. Let us emphasize that the two actions $S$ and $\tilde{S}$ live on different spaces and contain different fields, but are numerically equal. This is a consequence of the fact that the holographic relation between the fields $\phi$ and $\tilde{\phi}$ has been expressed as an invertible transform. The integration over $z$ can be evaluated using, say, Mathematica, to obtain it in the form of a sum of terms like

$$
\mathcal{P}\left(\tilde{x}, \tilde{x}^{\prime}\right) \sim \int|x-\tilde{x}|^{r}\left|x-\tilde{x}^{\prime}\right|^{s} G\left(\frac{|x-\tilde{x}|^{2}}{\left|x-\tilde{x}^{\prime}\right|^{2}}\right) d^{n-1} x
$$

with $r+s=2 \Delta-3 n+3$ and some function $G$. Exact expressions are given in the end. Hence, using (18), under the scaling $\tilde{x} \longrightarrow \lambda \tilde{x}$ accompanied by a change of variables $x \longrightarrow \lambda x, \mathcal{P}$ scales as $\mathcal{P}\left(\tilde{x}, \tilde{x}^{\prime}\right) \sim\left|\tilde{x}-\tilde{x}^{\prime}\right|^{2(1+\Delta-n)}$, and the action $\tilde{S}$ remains invariant. Consequently, the action $\tilde{S}$ on the boundary can be written as

$$
\tilde{S}=\frac{P_{0}}{L^{n}} \int \frac{\tilde{\phi}(\tilde{x}) \tilde{\phi}\left(\tilde{x}^{\prime}\right)}{\left|\tilde{x}-\tilde{x}^{\prime}\right|^{2(n-\Delta-1)}} d^{n-1} \tilde{x} d^{n-1} \tilde{x}^{\prime}
$$

where $P_{0}$ depends on the mass.

Let us now consider a self-interacting scalar field in the bulk and derive relations between the correlation functions of the bulk and the boundary theories. Adding a potential $V(\phi)$ to the bulk action (30) we consider

$$
S_{I}(\phi)=S(\phi)+S_{\mathrm{int}}(\phi),
$$

where $S_{\text {int }}(\phi)=\int d z d^{n-1} x V(\phi)$. The generating functional of the interacting theory,

$$
Z_{I}=\int D \phi e^{S_{I}(\phi)},
$$

can be expressed in terms of that of the non-interacting theory plus a source term. Introducing a source $J$ in the bulk we write

$$
S(\phi, J)=S(\phi)+\int \sqrt{g} J(z, x) \phi(z, x) d z d^{n-1} x .
$$

Then

$$
Z_{I}=\left.e^{S_{\mathrm{int}}\left(\frac{1}{\sqrt{g}} \frac{\delta}{\delta J}\right)} Z[J]\right|_{J=0},
$$

where

$$
Z[J]=\int D \phi e^{S(\phi, J)} .
$$

Correlation functions are computed as moments by differentiating $Z[J]$ with respect to the source. Using (31) and the transform (20) we rewrite the source term in $S(\phi, J)$ in terms of $\tilde{\phi}$ to obtain

$$
\tilde{S}(\tilde{\phi}, \tilde{J})=\tilde{S}(\tilde{\phi})+\int \tilde{J}(\tilde{x}) \tilde{\phi}(\tilde{x}) d^{n-1} \tilde{x}
$$

where we have defined

$$
\tilde{J}(\tilde{x})=\frac{\Phi_{0}(n, \Delta)}{L^{n-1}} \int \sqrt{g} \mathcal{K}(z, x \mid \tilde{x}) J(z, x) d z d^{n-1} x .
$$


Derivatives with respect to the sources are related by

$$
\frac{\delta}{\delta J^{\prime}(z, x)} \stackrel{\text { def }}{=} \frac{1}{\sqrt{g}} \frac{\delta}{\delta J(z, x)}=\frac{\Phi_{0}(n, \Delta)}{L^{n-1}} \int d^{n-1} \tilde{x} \mathcal{K}(z, x \mid \tilde{x})\left(\frac{\delta}{\delta \tilde{J}(\tilde{x})}\right) .
$$

Equality of the actions $S$ and $\tilde{S}$ then implies that the correlation functions computed in the bulk and in the boundary theories, respectively as normalized $J$-moments of $Z[J]$ and $\tilde{J}$-moments of $\tilde{Z}$, with the latter defined as

$$
\tilde{Z}[\tilde{J}]=\int D \tilde{\phi} e^{\tilde{S}(\tilde{\phi}, \tilde{J})}
$$

are equal.

Let us illustrate this with an example by considering the interaction potential $V(\phi)=\lambda \phi(z, x)^{k}$, for a fixed positive integer $k$. The $\ell$-th loop contribution to the twopoint function is

$$
\left\langle\phi\left(z_{1}, x_{1}\right) \phi\left(z_{2}, x_{2}\right)\right\rangle_{\ell}=\left.\frac{\lambda^{\ell}}{\ell !} \frac{\delta^{2}}{\delta J^{\prime}\left(z_{1}, x_{1}\right) \delta J^{\prime}\left(z_{2}, x_{2}\right)} \prod_{i=1}^{\ell} \int d z_{i}^{\prime} d^{n-1} y_{i} \frac{\delta^{k}}{\delta J^{\prime}\left(z_{i}^{\prime}, y_{i}\right)^{k}} Z[J]\right|_{J=0} .
$$

Using $\tilde{Z}[\tilde{J}]$ in place of $Z[J]$ and (41), we obtain

$$
\begin{aligned}
\left\langle\phi\left(z_{1}, x_{1}\right) \phi\left(z_{2}, x_{2}\right)\right\rangle_{\ell}= & \frac{\lambda^{\ell} \Phi_{0}^{k \ell+2}}{L^{(k \ell+2)(n-1)}} \int d^{n-1} \tilde{x}_{1} d^{n-1} \tilde{x}_{2} \prod_{i=1}^{\ell} \prod_{j=1}^{k} d z_{i}^{\prime} d^{n-1} y_{i} d^{n-1} \tilde{y}_{(i, j)} \\
& \mathcal{K}\left(z_{1}, x_{1} \mid \tilde{x}_{1}\right) \mathcal{K}\left(z_{2}, x_{2} \mid \tilde{x}_{2}\right) \mathcal{K}\left(z_{i}^{\prime}, y_{i} \mid \tilde{y}_{(i, j)}\right)\left\langle\tilde{\phi}\left(\tilde{x}_{1}\right) \tilde{\phi}\left(\tilde{x}_{2}\right) \prod_{\imath=1}^{\ell} \prod_{\jmath=1}^{k} \tilde{\phi}\left(\tilde{y}_{(i, j)}\right)\right\rangle,
\end{aligned}
$$

where

$$
\left\langle\tilde{\phi}\left(\tilde{x}_{1}\right) \tilde{\phi}\left(\tilde{x}_{2}\right) \cdots \tilde{\phi}\left(\tilde{x}_{m}\right)\right\rangle=\left.\prod_{i=1}^{m} \frac{\delta}{\delta \tilde{J}\left(\tilde{x}_{i}\right)} \tilde{Z}[\tilde{J}]\right|_{\tilde{J}=0}
$$

denotes the $m$-point function of the boundary theory. This feature generalizes. Generally, the $\ell$-th loop contribution to the $p$-point function in the bulk is determined by the $(k \ell+p)$ point function of the boundary theory.

To conclude, we have obtained the HKLL bulk reconstruction formula as the Gel'fandGraev-Radon transform on the $n$-dimensional Euclidean anti-de Sitter space for $n>1$. This is achieved with the assumption (19) that the transform on the positive light cone is given by a conformal field on the boundary. This allows us to relate the scalar field actions in the bulk and that at the boundary. While expressed in terms of the transformed fields the actions are numerically equal. We use this to write the generating functionals in the two theories, incorporating interactions in the bulk. It is then showed that for a $\phi^{k}$ interaction term in the bulk the $p$-point correlation function at the $\ell$-th loop is related to the $(p+k \ell)$-point correlation function in the boundary, in keeping with holography.

Integral geometry has been discussed earlier in the context of AdS-CFT duality. In one approach the CFT was associated to a Fourier transform [22], which in turn was related 
to the Gel'fand-Graev technique. In another approach [23] OPE blocks on the kinematic space defined by the configuration space of two points on $\mathrm{AdS}_{3}$, was considered. The kinematic space, having double the dimension, is looked upon as the space of geodesics in the bulk ending on the pair of points at the boundary. Geodesic Radon transform was then used to interpret the OPE blocks in the bulk as "geodesic operators", relating the correlators in terms of the length of geodesics in the bulk. The approach we have taken in here is a more direct one. We consider the boundary of $\mathrm{AdS}_{n}$ and identify it on the positive light cone. Then, an inverse Radon transform, in conjunction with the assumption of conformality on the light cone (19) is used to obtain the bulk fields. The assumption of conformality on $\mathscr{C}_{n}^{+}$restricts the space of distributions on the boundary, which otherwise may be taken to be the Schwartz space. Indeed, it is because of this assumption that the otherwise ill-defined normalization constant $c_{n}$ is multiplied with an extra term to make the coefficient of the transform finite, as in (26). We believe that this computation will be useful in understanding the structure of the bulk reconstruction procedure.

Computation of $c_{\boldsymbol{n}}$ in (9). In order to compute the constant $c_{n}$ it is convenient to first write (8) after integrating on $X$ as

$$
I=\int_{\mathcal{M}} \int_{\mathscr{C}_{n}^{+}} \frac{\delta\left(\eta_{a b} X^{a} \xi^{b}+p\right)}{\left|\eta_{a b} Y^{a} \xi^{b}+p\right|^{n}} d^{n} \xi d^{n} X
$$

We shall not perform the integration over $X$, but keeping it in the integral is useful to keep track of factors occurring in change of variables. Interpreting the integral as

$$
I=\int_{\mathcal{M}}\left(\int_{\mathscr{C}_{n}^{+} \cap \mathscr{S}_{n-1}} \frac{1}{\left|\eta_{a b} Y^{a} \xi^{b}+p\right|^{n}} d^{n} \xi\right) d^{n} X
$$

we perform the integration over $\xi$ by restricting $d \xi^{0} d^{n-1} \tilde{x}$ to $\mathscr{S}_{n-1}$ as $d^{n-1} \tilde{x} / \frac{\partial \Sigma}{\partial \xi^{0}}$, with $\xi^{0}$ evaluated in terms of $\tilde{x}$ from (17). Moreover, we substitute the affine coordinates (10) for $X$ and $Y$ as $(z, x)$ and $\left(w, x^{\prime}\right)$, respectively along with (13) in the integral. We first define new variables as

$$
z=w \tau, \quad x=w \hat{x}, \quad x^{\prime}=w \widehat{x^{\prime}}, \quad \tilde{x}=w \widehat{\tilde{x}} .
$$

Then with a further change of variables

$$
\hat{x}=\widehat{\tilde{x}}+y, \quad \hat{x}-\widehat{x^{\prime}}=r,
$$

we rewrite the integral as

$$
I=\frac{2^{n-1}}{L \alpha^{2}} \int \frac{\left(\tau^{2}+y^{2}\right)}{\left|\tau^{2}+y^{2}-\tau\left(1+(y-r)^{2}\right)\right|^{n}} \frac{d \tau}{\tau} d^{n-1} r d^{n-1} y .
$$

Using the symmetries of $\mathcal{M}$ we now set $r=0$ in the integrand [18], replacing the integration over $r$ with the volume of the $(n-1)$-dimensional sphere, $V_{n-1}=\frac{2 \pi^{(n-1) / 2}}{\Gamma((n-1) / 2)}$. Defining further, $y=\sqrt{\tau} \rho$, the integral finally assumes the form

$$
I=\int_{0}^{1}\left[\frac{2^{n} \pi^{(n-1) / 2} V_{n-1}}{\alpha^{2} L \Gamma((n-1) / 2)}\left(\int_{0}^{1} \frac{\left(1+\rho^{2}\right) \rho^{n-2}}{\left(1-\rho^{2}\right)^{n}} d \rho\right)\left(\frac{(1+\tau)\left(1+\tau^{2 n-2}\right)}{(1-\tau)^{n}} \tau^{-(n+1) / 2}\right)\right] d \tau,
$$


Evaluating the integration over $\rho$ it can be seen that the integrand inside the square braces is supported at $\tau=1$, which corresponds to $z=w$. The integrand is thus a delta function, as in (8), with strength $1 / c_{n}$, with $c_{n}$ given by (9).

Constants appearing in the action. The kernel $\mathcal{P}\left(\tilde{x}, \tilde{x}^{\prime}\right)$ in (31) is

$$
\mathcal{P}\left(\tilde{x}, \tilde{x}^{\prime}\right)=\int\left(P_{1}\left(x, \tilde{x}, \tilde{x}^{\prime}\right)+\sum_{i=1}^{n-1}\left(a^{i} b^{i}\right) P_{2}\left(x, \tilde{x}, \tilde{x}^{\prime}\right)+m^{2} P_{3}\left(x, \tilde{x}, \tilde{x}^{\prime}\right)\right) d^{n-1} x
$$

where we have defined $a^{i}=x^{i}-\tilde{x}^{i}$ and $b^{i}=x^{i}-\tilde{x}^{\prime i}$. The three functions are written in terms of the Gaussian hypergeometric function $F$ as

$$
\begin{aligned}
& P_{1}\left(x, \tilde{x}, \tilde{x}^{\prime}\right)=a^{-2 n+2 \Delta+2} b^{1-n}\left(3+n^{2}-4 n\right) \frac{\Gamma\left(\frac{n-3}{2}\right) \Gamma\left(\frac{n-2 \Delta-1}{2}\right)}{8 \Gamma(n-\Delta)} F\left(\frac{n-2 \Delta-1}{2}, n-\Delta ; \frac{1-n}{2} ; \frac{b^{2}}{a^{2}}\right) \\
& +\left(a^{-2 n+2 \Delta+2} b^{1-n}+a^{2 \Delta-2 n} b^{3-n}\right)\left(4 n+2 \Delta(n-3)-n^{2}-3\right) \frac{\Gamma\left(\frac{n-3}{2}\right) \Gamma\left(\frac{n-2 \Delta-1}{2}\right)}{8 \Gamma(n-\Delta)} F\left(\frac{n-2 \Delta+1}{2}, n-\Delta ; \frac{3-n}{2} ; \frac{b^{2}}{a^{2}}\right) \\
& \quad+a^{2 \Delta-2 n} b^{3-n}\left(n^{2}+4 \Delta^{2}-4 n \Delta-1\right) \frac{\Gamma\left(\frac{n-3}{2}\right) \Gamma\left(\frac{n-2 \Delta-1}{2}\right)}{8 \Gamma(n-\Delta)} F\left(\frac{n-2 \Delta+3}{2}, n-\Delta ; \frac{5-n}{2} ; \frac{b^{2}}{a^{2}}\right) \\
& \quad-\left(a^{-3 n+2 \Delta+1} b^{2}+a^{-3 n+2 \Delta+3}\right)\left(3 n^{2}-2 \Delta-2 n \Delta-3\right) \frac{\Gamma\left(-\frac{n+1}{2}\right) \Gamma\left(\frac{3(n-1)}{2}-\Delta\right)}{8 \Gamma(n-\Delta)} F\left(\frac{3 n-2 \Delta-1}{2}, n-\Delta ; \frac{n+1}{2} ; \frac{b^{2}}{a^{2}}\right) \\
& \quad+a^{-3 n+2 \Delta+1} b^{2}\left(3+9 n^{2}+4 \Delta^{2}+8 \Delta-12 n \Delta-14 n\right) \frac{\Gamma\left(-\frac{n+1}{2}\right) \Gamma\left(\frac{3(n-1)}{2}-\Delta\right)}{8 \Gamma(n-\Delta)} F\left(\frac{3 n-2 \Delta+1}{2}, n-\Delta ; \frac{n+3}{2} ; \frac{b^{2}}{a^{2}}\right) \\
& \quad-a^{-3 n+2 \Delta+3}\left(n^{2}-1\right) \frac{\Gamma\left(-\frac{n+1}{2}\right) \Gamma\left(\frac{3(n-1)}{2}-\Delta\right)}{8 \Gamma(n-\Delta)} F\left(\frac{3 n-2 \Delta-3}{2}, n-\Delta ; \frac{n-1}{2} ; \frac{b^{2}}{a^{2}}\right) \\
& P_{2}\left(x, \tilde{x}, \tilde{x}^{\prime}\right)=a^{2 \Delta-3 n+1} \frac{\Gamma\left(\frac{1-n}{2}\right) \Gamma\left(\frac{3 n-2 \Delta-1}{2}\right)}{2 \Gamma(n-\Delta)} F\left(\frac{3 n-2 \Delta-1}{2}, n-\Delta ; \frac{n+1}{2} ; \frac{b^{2}}{a^{2}}\right) \\
& \quad+a^{2 \Delta-2 n} b^{1-n} \frac{\Gamma\left(\frac{n-2 \Delta+1}{2}\right) \Gamma\left(\frac{n-1}{2}\right)}{2 \Gamma(n-\Delta)} F\left(\frac{n-2 \Delta+1}{2}, n-\Delta ; \frac{3-n}{2} ; \frac{b^{2}}{a^{2}}\right) \\
& P_{3}\left(x, \tilde{x}, \tilde{x}^{\prime}\right)=a^{2 \Delta-3 n+3} \frac{\Gamma\left(\frac{3 n-2 \Delta-3}{2}\right) \Gamma\left(\frac{1-n}{2}\right)}{2 \Gamma(n-\Delta-1)} F\left(\frac{3 n-2 \Delta-3}{2}, n-\Delta-1 ; \frac{n+1}{2} ; \frac{b^{2}}{a^{2}}\right) \\
& \quad+a^{2 \Delta-2 n+2} b^{1-n} \frac{\Gamma\left(\frac{n-2 \Delta-1}{2}\right) \Gamma\left(\frac{n-1}{2}\right)}{2 \Gamma(n-\Delta-1)} F\left(\frac{n-2 \Delta-1}{2}, n-\Delta-1 ; \frac{3-n}{2} ; \frac{b^{2}}{a^{2}}\right)
\end{aligned}
$$

The formulas are written in the least cluttered form. The Gamma functions are to be analytically continued depending on the values of $n$ and $\Delta$.

\section{Acknowledgments}

SB and KR thank Bobby Ezhuthachan for illuminating discussions on various occasions. 
Open Access. This article is distributed under the terms of the Creative Commons Attribution License (CC-BY 4.0), which permits any use, distribution and reproduction in any medium, provided the original author(s) and source are credited.

\section{References}

[1] J.M. Maldacena, The large- $N$ limit of superconformal field theories and supergravity, Int. J. Theor. Phys. 38 (1999) 1113 [hep-th/9711200] [INSPIRE].

[2] E. Witten, Anti-de Sitter space and holography, Adv. Theor. Math. Phys. 2 (1998) 253 [hep-th/9802150] [INSPIRE].

[3] S.S. Gubser, I.R. Klebanov and A.M. Polyakov, Gauge theory correlators from noncritical string theory, Phys. Lett. B 428 (1998) 105 [hep-th/9802109] [INSPIRE].

[4] V. Balasubramanian, S.B. Giddings and A.E. Lawrence, What do CFTs tell us about Anti-de Sitter space-times?, JHEP 03 (1999) 001 [hep-th/9902052] [INSPIRE].

[5] I. Bena, On the construction of local fields in the bulk of $A d S_{5}$ and other spaces, Phys. Rev. D 62 (2000) 066007 [hep-th/9905186] [INSPIRE].

[6] A. Hamilton, D.N. Kabat, G. Lifschytz and D.A. Lowe, Local bulk operators in AdS/CFT: A boundary view of horizons and locality, Phys. Rev. D 73 (2006) 086003 [hep-th/0506118] [INSPIRE].

[7] A. Hamilton, D.N. Kabat, G. Lifschytz and D.A. Lowe, Holographic representation of local bulk operators, Phys. Rev. D 74 (2006) 066009 [hep-th/0606141] [INSPIRE].

[8] A. Hamilton, D.N. Kabat, G. Lifschytz and D.A. Lowe, Local bulk operators in AdS/CFT: A holographic description of the black hole interior, Phys. Rev. D 75 (2007) 106001 [Erratum ibid. D 75 (2007) 129902] [hep-th/0612053] [INSPIRE].

[9] D. Kabat, G. Lifschytz, S. Roy and D. Sarkar, Holographic representation of bulk fields with spin in AdS/CFT, Phys. Rev. D 86 (2012) 026004 [arXiv:1204.0126] [INSPIRE].

[10] D. Kabat and G. Lifschytz, Decoding the hologram: Scalar fields interacting with gravity, Phys. Rev. D 89 (2014) 066010 [arXiv:1311.3020] [InSPIRE].

[11] D. Kabat and G. Lifschytz, Bulk equations of motion from CFT correlators, JHEP 09 (2015) 059 [arXiv: 1505. 03755] [INSPIRE].

[12] S.R. Roy and D. Sarkar, Hologram of a pure state black hole, Phys. Rev. D 92 (2015) 126003 [arXiv: 1505.03895] [INSPIRE].

[13] D. Kabat and G. Lifschytz, Local bulk physics from intersecting modular Hamiltonians, JHEP 06 (2017) 120 [arXiv:1703.06523] [INSPIRE].

[14] F. Sanches and S.J. Weinberg, Boundary dual of bulk local operators, Phys. Rev. D 96 (2017) 026004 [arXiv: 1703.07780] [INSPIRE].

[15] K. Goto and T. Takayanagi, CFT descriptions of bulk local states in the AdS black holes, arXiv: 1704.00053 [INSPIRE].

[16] S.R. Roy and D. Sarkar, Holographic bulk reconstruction beyond (super)gravity, arXiv:1704.06294 [INSPIRE].

[17] G. Sárosi and T. Ugajin, Modular Hamiltonians of excited states, OPE blocks and emergent bulk fields, arXiv:1705.01486 [INSPIRE]. 
[18] I.M. Gel'fand and M.I. Graev, Generalized Functions: Integral geometry and representation theory, Generalized functions, Academic Press (1966).

[19] S. Helgason, Integral Geometry and Radon Transforms, Springer (2011).

[20] G. Solanes, Integral geometry and curvature integrals in hyperbolic space, http://mat.uab.es/ egallego/gil/tesiEng.pdf.

[21] S. Rychkov, EPFL Lectures on Conformal Field Theory in $D \geq 3$ Dimensions, SpringerBriefs in Physics, Springer (2017) [arXiv: 1601.05000] [INSPIRE].

[22] I.M. Tolfree, An Alternative Path to the Boundary: The CFT as the Fourier Space of AdS, Phys. Rev. D 78 (2008) 106002 [arXiv:0809.0485] [INSPIRE].

[23] B. Czech, L. Lamprou, S. McCandlish, B. Mosk and J. Sully, A Stereoscopic Look into the Bulk, JHEP 07 (2016) 129 [arXiv: 1604.03110] [INSPIRE]. 